\title{
Kulturelle Anatomie
}

\author{
Erhard Taverna
}

Migrantenspezifische Probleme im medizinischen Alltag gehören in jedem Einwanderungsland zur Fortbildung. Das erinnert daran, dass die Körperwahrnehmung auch sogenannter Fachpersonen durch die eigene kulturelle Tradition, persönliche Biographie und eigenes Geschlecht entscheidend beeinflusst wird. Bekannte Beispiele dafür sind Themen wie: Medikamente und Nebenwirkungen bei Frauen, Herzinfarkte bei Frauen, geburtshilfliche Methoden oder Hormonersatztherapien in der Menopause. Es gibt einen «gender bias», der immer wieder für medizinische Überraschungen sorgt. Vor allem in einem Land, das sich mit Frauenfragen notorisch schwertut.

Der Blick in den Anatomieatlas schliesst blinde Flecken nicht aus. Wer ihn erfolgreich nur als Werkanleitung und Bastelbogen umsetzt, kann andere Realitäten wie Rollenbilder, Sehgewohnheiten und Politik ausblenden. Die Folgen sind falsche Indikationen und schlechte Resultate, in jedem Fall eine Verschleuderung von Ressourcen.

$\mathrm{Ab}$ und zu kann es nicht schaden, die Sehweisen fachfremder Spezialisten kennenzulernen. Zum Beispiel das Buch einer wissenschaftlichen Assistentin mit einem Faible für Körperinszenierungen und einem Professor, der sich für historische Anthropologie interessiert. Beide entstammen, wie die Mitautoren, einem breiten disziplinären Feld, das sich mit der historischen Erforschung von Körperlichkeit befasst. Es sind viele Wissenschaften vertreten, ausgenommen die somatische Medizin: Kultur, Kunst und Literatur, Philosophie, Erziehung, Soziologie, Geschichte, Ethnologie, Archäologie, Linguistik, Semiotik und Psychoanalyse. Die traditionellübliche, medizinhistorische Sichtweise wird durch die neuen Fragestellungen aufgebrochen.
Diese transdisziplinäre Bluttransfusion belebt ein anämisches Randgebiet, das normalerweise in der Praxis überhaupt keine Rolle spielt. Die Analyse kultureller Formen der Teilung und Ordnung des Körpers korrigiert die eigene, oft verzerrte Optik, in dem sie, nicht nur dem Mediziner, eigene Defizite und unbewusste Vorurteile sichtbar macht.

Es geht immer um Körperteile, um neuzeitliche Fragmentierungen, die fortschreitende Prothetisierung, die Vernetzung mit Maschinen, um die Zerstückelungswut im modernen Kino und Theater. Bilder von René Magritte wie «Der magische Spiegel» oder «Die Evidenz» symbolisieren, dass es keinen menschlichen Körper in einem absoluten Sinn gibt. Ein kunstgeschichtlicher Abriss zu einem abwesenden Teil des weiblichen Körpers in der bildenden Kunst, dieser Leerstelle zwischen den Beinen, wäre als Vorlesung jeder medizinischen Fakultät zu empfehlen. Jedes Kapitel ist informativ, spannend und vergnüglich zu lesen, egal ob es sich um den Hintern in der Antike, psychoanalytische Phalluskonstruktionen, die verrutschte Vulva in Kultfilmen wie «Matrix», die Bildgeschichte des Bauches in der Renaissance oder um die seltsamen Verzückungen christlicher Nonnen handelt. Die 24 Beiträge liefern Anatomisches von Kopf bis Fuss, sie erfassen das Schöne und Kostbare genauso wie das Grausame, Hässliche, Eklige und Banale. Der röhrenförmige Blick des Spezialisten weitet sich zum gesellschaftlichen Panorama. Allein schon aus diesem Grund kann das Werk als gute Ergänzung zum bisherigen Fachunterricht empfohlen werden.

- Benthien C, Wulf C (Hrsg.). Körperteile, eine kulturelle Anatomie. rowohlts enzyklopädie, Taschenbuch Verlag; 2001, 523 Seiten. 